\section{[gw22-e0994] THROUGH IVUS STUDY OF CHARACTERISED THE RELATIONSHIP AS STABLE ANGINA (SA) AND UNSTABLE ANGINA (UA) PATIENTS}

Hai-jian Su, Ming Zhang Liaoning University of Traditional Chinese Medicine, Liaoning, China

\subsection{6/heartjnl-2011-300867.499}

Purpose As medical technology advances, the understanding of coronary heart disease from the previous clinical diagnosis, diagnostic ECG, ECG to diagnosis, then diagnostic coronary angiography (coronary angiography $\mathrm{CAG}$ ), and then by coronary angiography (CAG) to the coronary intravascular ultrasound (intravascular ultrasound IVUS) diagnostic level to a higher level. Intravascular ultrasound (IVUS) can provide high resolution within the lumen of coronary lumen and plaque cross-sectional images of coronary plaque can also provide detailed information and the wall (including the plaque volume, composition, with or without calcification and calcification of the situation is stable, with or without rupture, and vessel wall volume and shape, etc), so the study of coronary atherosclerosis as a powerful tool, through the stable angina pectoris (stable angina Peetori SAP) and unstable angina (unstable angina Peetori, UAP) in patients with coronary artery coronary angiography (CAG) and intravascular ultrasound (IVUS) examination, and images images study with stable angina (SA) and unstable angina (UA) vascular remodeling in patients with coronary artery disease, coronary artery disease and coronary artery calcified plaque characterised the relationship as stable angina (SA) and unstable angina (UA) patients prevention and treatment of plaque made reliable basis. Materials and methods From January 2010 to December 2010 collected 60 cases of coronary heart disease patients with stable angina pectoris (SAP), unstable angina pectoris (UAP) in the diagnostic criteria with reference to the 1979 WHO diagnostic criteria established coronary heart disease and 2001, the Chinese Society of Cardiology and the Medical Journal of Cardiology Editorial Board to develop a 'diagnosis of unstable angina and treatment recommendations', the latest American Heart Association and European Society of Cardiology Association (AHA, ESC) technical guidelines established coronary heart disease treatment for stable angina (SA) group of 30 patients, mean age $60.75 \pm 9.06$ years old, 24 males and 6 females; unstable angina (UA) group of 30 patients, mean age $51.56 \pm 8.02$ years old, 16 males and 14 females, early onset exertional angina in 14 cases, worsening exertional anginatype 16 patients. CAG checks were carried out two groups of patients, while application of IVUS disease in two groups of ischemia-related inspection, the target lesion by clinical, ECG and coronary angiography to help determine. The use of IVUS measurements, calculated at the smallest vessels of the lesion external elastic membrane area (EEMA), lumen area (LA), plaque area (team), plaque burden, lipid pool area, lipid pool/ plaque area, lipid pool/plaque area, fibrous cap thickness, calcification of curvature, calcification range, calcification type.

Results (1) SA group of stable plaque lesion than at the UA group (40, 91.0\% vs 5, 14.3\%, p <.0.05),; UA vascular lesions were sentenced to more than the SA group with unstable plaque $(30,40.0 \%$ vs $5,16.7 \%, p<0.05)$. SA group lesion at or slightly eccentric to the concentric plaque than UA group (33, $75.0 \%$ vs 7 , $20.0 \%, p<0.05)$; UA group of vascular lesions at the more eccentric plaque lipid in the SA group (20,57.1\% vs 8, $18.1 \%$, p <0.05). SA group hyperechoic lesion at the fibrous plaque as the cap $(1.11 \pm 0.75 \mathrm{~mm})$, vascular lesions than the UA group at the thin fibrous cap $(0.51 \pm 0.33 \mathrm{~mm})$ thick, $\mathrm{p}<0.05$, significant difference.
(2) SA and UA patients the culprit lesions in patients with different degrees of calcification were loading, SA group of large and small lesion calcification at more than UA group $(25,66.8 \%$ vs $5,11.2 \%, p<0.05)$, UA lesions were few and small vessels were calcified than SA group (15, 42.9\% vs 8, 18.2\%, p <0.05). (3) SA group of patients with vascular lesions at the UA group than negative remodeling $(33,75.0 \%$ vs $5,14.3 \%$, $p<0.05)$; UA Department vascular lesions in patients, more than the SA group is reconstructed $(25,71.4 \%$ compared with $7,16.0 \%, p<0.05)$.

Conclusion (1) Stable angina pectoris (SAP) in patients with stable plaque lesion mainly fine, more performance for the concentric or eccentric, etc., or high light echo of fibrous plaque; unstable angina pectoris (UAP) vascular lesions in patients with unstable plaques mainly fine, more performance for the eccentricity of the lipid plaque, a large lipid pool, thin fibrous cap. (2) Stable angina pectoris (SAP) and unstable angina patients (UAP) in patients with lesions of different degrees of calcification were the load. SAP and fewer patients with large calcified lesions, UAP patients have few and small, calcified lesions. (3) Stable angina pectoris (SAP) lesions were mainly negative vascular remodeling at the main; unstable angina pectoris (UAP) lesions were positive and negative vessels were mainly for the reconstruction of the main. 